\title{
The impact of the COVID-19 pandemic on positive airway pressure usage in children with sleep-disordered breathing
}

\author{
Kanokkarn Sunkonkit ${ }^{1,2,3} \cdot$ Sarah Selvadurai ${ }^{4}$. Giorge Voutsas ${ }^{3,4} \cdot$ David Benzon $^{4} \cdot$ Adele Baker $^{1} \cdot$ Melissa Trinh $^{1}$. \\ Indra Narang ${ }^{1,3,4}$
}

Received: 23 April 2021 / Revised: 16 May 2021 / Accepted: 18 May 2021 / Published online: 9 June 2021

(c) The Author(s), under exclusive licence to Springer Nature Switzerland AG 2021

\begin{abstract}
Purpose To evaluate the impact of the COVID-19 pandemic on non-invasive positive airway pressure (PAP) usage among children with sleep-disordered breathing (SDB).

Methods PAP usage data in children with SDB aged 1 to 18 years old at The Hospital for Sick Children, Canada, were analyzed. The PAP usage data were recorded for 3 months prior to and 3 months following the COVID-19 lockdown in Ontario, Canada. The primary outcomes of interest were (i) percentage of days that PAP was used for $\geq 4 \mathrm{~h}$ and (ii) average daily usage of PAP based on days when PAP was used.

Results A total of 151 children were included. The mean $( \pm \mathrm{SD})$ age and BMI were $12.6 \pm 4.1$ years and $28.7 \pm 12.4 \mathrm{~kg} / \mathrm{m}^{2}$, respectively. The median (IQR) percentage of days of PAP usage for $\geq 4 \mathrm{~h}$ and average nightly PAP usage was significantly higher during compared with prior to the pandemic (76.7 [19.0-94.0] vs 62.0 [15.5-89.0]\%, $p=0.02$, and 406.0 [244.0-525.0] vs 367.0 [218.0-496.0] min, $p=0.006$, respectively). Within this cohort, 95/151 (63\%) children with SDB showed increased PAP usage and 56/151 (37\%) either decreased the amount of time they used PAP or stopped PAP use altogether.

Conclusions COVID-19 pandemic has provided opportunities for increased PAP usage in a significant number of children with SDB. A subset of children with prior evidence for suboptimal PAP usage showed further decreases in PAP usage during the pandemic. This information is critical for clinicians to provide anticipatory guidance to encourage PAP usage both during the pandemic and beyond.
\end{abstract}

Keywords Coronavirus disease 2019 (COVID-19) pandemic · Positive airway pressure therapy · Adherence · Sleepdisordered breathing $\cdot$ Pediatrics

\section{Introduction}

Sleep-disordered breathing (SDB) encompasses various respiratory or ventilatory abnormalities occurring during

Kanokkarn Sunkonkit and Sarah Selvadurai share first authorship.

Indra Narang

indra.narang@sickkids.ca

1 Division of Respiratory Medicine, The Hospital for Sick Children, 555 University Ave, Toronto, ON M5G 1X8, Canada

2 Division of Pulmonary and Critical Care, Department of Pediatrics, Faculty of Medicine, Chiang Mai University, Chiang Mai, Thailand

3 University of Toronto, Toronto, Canada

4 Translational Medicine, Research Institute, The Hospital for Sick Children, Toronto, Canada sleep including obstructive and central sleep apnea [1]. Obstructive sleep apnea (OSA) is characterized by snoring and recurrent partial and/or complete obstruction of the upper airway during sleep [1] which affects $1-4 \%$ of otherwise healthy children [2] and $25-80 \%$ of children and adolescents who are obese and/or have underlying medical comorbidity such as trisomy 21 and Chiari malformation [3-5]. Central sleep apnea (CSA) is characterized by the absence of central respiratory drive during sleep, resulting in abnormal gas exchange and inadequate ventilation [6]. Specifically, CSA may coexist with nocturnal hypoventilation, which is defined as an end-tidal $\mathrm{CO}_{2}\left(\mathrm{etCO}_{2}\right)$ and/or transcutaneous $\mathrm{CO}_{2}$ $\left(\mathrm{tcCO}_{2}\right)>50 \mathrm{mmHg}$ for more than $25 \%$ of total sleep time [6]. Untreated SDB results in adverse outcomes including behavioral problems [7], lower school performance [8], increased cardiovascular and metabolic risk [9], as well as depression [10]. 
Children with complex chronic conditions were at higher risk of having residual OSA after adenotonsillectomy (AT) [11] although AT is the recommended first-line treatment for OSA [12]. Persistent OSA post AT and/or CSA and nocturnal hypoventilation are typically treated with positive airway pressure (PAP) therapy, which henceforth refers to non-invasive ventilation such as continuous positive airway pressure (CPAP) or bi-level PAP [13, 14].

Consistent nightly use of PAP is important as PAP is associated with several health benefits including better mood, improved quality of life, improved school performance, and reductions in cardiometabolic risk [14-16]. PAP is an effective treatment for SDB when worn nightly; however, low PAP adherence limits the potential benefits of PAP therapy $[17,18]$.

The COVID-19 pandemic is an ongoing viral pandemic of a novel infectious disease caused by severe acute respiratory syndrome coronavirus 2 (SARS-CoV-2). During the pandemic, there are concerns regarding the use of PAP as the World Health Organization (WHO) listed PAP therapy as a high-risk aerosol-generating medical procedure (AGMP) that may cause COVID-19 transmission $[19,20]$. During the initial period of the pandemic of COVID-19, healthcare workers advocated withholding the use of PAP unless medically necessary [21]. Consequently, there were significant concerns among caregivers regarding PAP use in the home especially among children who were obese and with underlying medical comorbidities who were deemed to be at high risk of COVID-19 infection and therefore could potentially spread COVID-19 to household members [21].

Currently, there are no data on PAP adherence before and during the COVID pandemic in the pediatric population. These data are crucial to ensure that PAP usage has not further declined during the pandemic. Such information may also be beneficial for designing and implementing targeted interventions to sustain and improve PAP adherence during as well as beyond the pandemic. Thus, the aim of this study was to compare changes in PAP adherence rates among children with SDB before and during the COVID-19 pandemic in Ontario, Canada.

\section{Materials and methods}

\section{Study population}

This was a retrospective study with children, aged $1-18$ years of age, diagnosed with SDB who were prescribed PAP therapy at The Hospital for Sick Children (Toronto, Canada). PAP usage were evaluated in children before and during the COVID-19 pandemic, and children were included if they were prescribed PAP for SDB at home for a minimum of
1 month and up to 3 months during two defined time periods: (1) prior to the pandemic in Ontario, Canada (December 18, 2019-March 16, 2020), and (2) during the pandemic (March 17-June 14, 2020), which immediately followed the COVID-19 lockdown, declared on March 17, 2020, in Ontario, Canada. The COVID-19 lockdown enforced provincial measures to reduce COVID-19 transmission including a stay-at-home order except for essential trips (e.g., groceries, medical appointments) and social distancing. Children were excluded if (1) PAP therapy was only prescribed during the COVID-19 lockdown and/or (2) PAP data was not available during both time periods as specified above. Electronic medical records were reviewed, and the data recorded that were included were baseline demographics and anthropometrics (age, gender, height, weight, body mass index (BMI)), medical history which included primary diagnosis, type of SDB, comorbidities, history of AT, whether the child was an independent PAP user (e.g., able to apply and use PAP at home independently of parental/caregiver support), allergies, medications, type of PAP therapy, duration of initiation of PAP therapy, and PAP adherence data. Obesity was defined as a BMI greater than or equal to the $95^{\text {th }}$ percentile for sex and age [22].

\section{PAP adherence}

Objective data on usage of PAP therapy was assessed via device data reports obtained remotely. As all participants used ResMed PAP devices, the software used to assess adherence included AirView (ResMed; San Diego, CA, USA) and ResScan for bi-level PAP (ResMed; San Diego, CA, USA) [23]. AirView, a securely hosted cloud-based system, is a password-protected cloud technology compliant with the Health Insurance Portability and Accountability Act requiring written informed consent or digital consent obtained by the medical equipment provider from the patient or their legal guardian. Data derived from the ResMed PAP device is recorded nightly and automatically uploaded via cellular connectivity to AirView on a daily basis, thereby allowing the medical equipment provider to remotely monitor patient adherence. ResScan is a password-protected data management software program allowing manual acquisition of ResMed PAP device data via USB or direct cable connection by clinician or medical equipment provider.

The main outcomes were (i) percentage of days where PAP was used for $\geq 4$-h usage (\%) and (ii) average nightly usage (minutes) defined as the average minutes of PAP use based on only the days where PAP was used. Additional measures included the overall percentage of days that PAP was used (regardless of duration of usage) and the average total usage (minutes) defined as the average minutes of PAP use based on all the days over which the data was collected. 


\section{Statistical analysis}

Data were analyzed with SPSS version 26.0 (IBM Corp, Armonk, NY). Descriptive statistics, including frequencies of and percentages for categorical variables, and mean $( \pm S D)$ for continuous variables were computed for baseline characteristics and PAP parameters. PAP adherence was compared before and during the COVID-19 lockdown using the Wilcoxon signed-rank test for paired samples or paired $t$ test based on data distribution. Mixed-design repeated measures ANOVA subgroup analyses were also performed to compare PAP adherence data prior to and during the lockdown with between-subject factors including sex, diagnoses (e.g., obesity or underlying medical disorders such as trisomy 21, Chiari malformation, and other genetic diseases), age groups (e.g., younger than 12 years old, between 12 and 15 years old, and 15 years or older), and whether the child was an independent user. Baseline characteristics were also compared between subgroups across time periods using chisquare test for categorical variables and one-way ANOVA for continuous variables. Bonferroni post hoc testing method was performed for statistically significant differences to adjust for multiple comparisons. Subgroup ANOVA analyses were adjusted for the number of days of PAP download data collection prior to the COVID-19 lockdown. Comparisons in baseline characteristics between children who showed a decrease in PAP use and those who showed an increase in PAP use during the lockdown were performed using the chi-square test for categorical variables and the Mann-Whitney $U$ test for continuous variables. The degree of change in PAP adherence data from prior to lockdown was also compared between different subgroups using the Mann-Whitney $U$ test or Kruskal-Wallis $H$ test. Additional analyses included multiple linear regressions to evaluate the association between the percentage of days with a minimum 4-h PAP usage and average nightly PAP usage as the main outcomes, and baseline characteristics (e.g., age, diagnoses, sex, race, PAP type, independent or dependent user) as well as the number of days of PAP download data collection prior to the COVID-19 lockdown.

\section{Ethics}

The present study was approved by the Research Ethics Board at The Hospital for Sick Children (REB \# 1,000,071,964), Toronto, Canada.

\section{Results}

A total of 151 children with SDB was included in this study (Table 1$)$. The mean $( \pm$ SD) age and BMI of the study population was $12.6 \pm 4.1$ years and $28.7 \pm 12.4 \mathrm{~kg} /$ $\mathrm{m}^{2}$, respectively, and 92/151 (60.9\%) were male. The mean $( \pm \mathrm{SD})$ duration of PAP therapy was $3.3 \pm 2.7$ years. Of the 151 children, 53 (35.1\%) were obese with no other coexisting medical conditions; $96(63.6 \%)$ had an at least one underlying medical condition (e.g., Chiari malformation, trisomy 21 ) and the remaining $2(1.3 \%)$ were otherwise healthy with no underlying medical diagnosis. In the cohort, $58.3 \%$ had a previous AT and $34.4 \%$ were independent PAP users. Additionally, $67.5 \%$ were prescribed among CPAP and $32.5 \%$ were prescribed bi-level PAP.

PAP download data were collected over a mean $( \pm$ SD) period of $87 \pm 12$ days and $89 \pm 5$ days prior to and during the lockdown, respectively, and all the data are shown in Table 2. For the entire cohort, the median (IQR) percentage of PAP usage for a minimum of $4 \mathrm{~h}$ increased $(p=0.02)$ during the lockdown when compared to prior to the lockdown period. Similarly, average nightly usage on days where PAP was used increased $(p=0.006)$, with a higher percentage of days where PAP was used $(p=0.03)$ as well as average total usage measured over the period that PAP data was collected $(p=0.03)$.

However, on further exploration, 95/151 (63\%) increased usage of PAP and 56/151 (37\%) showed a decrease in the usage of PAP during the COVID-19 pandemic lockdown period as compared to prior to the lockdown. Moreover, in those within the decreased PAP usage group, 11/56 (19.6\%) or 7.3\% of the entire group abruptly terminated PAP use during the lockdown and did not resume PAP during the remainder of data collection period. Of these 11 children, $8(73 \%)$ had at least one underlying medical condition and $3(27 \%)$ were obese with no other co-existing medical conditions. Comparing the increased and decreased PAP usage groups, there were no significant differences in baseline characteristics, including age, BMI, race, whether or not an independent PAP user, medical diagnoses, type of SDB, and PAP type (Table 3).

Specifically, the degree of change in PAP adherence from prior to lockdown was compared between the increased and decreased PAP usage groups (Table 4). Children in the decreased PAP usage demonstrated a greater median (IQR) percent change in the percentage of PAP usage for a minimum of $4 \mathrm{~h}(p<0.001)$, average nightly usage $(p<0.001)$, percentage of days where PAP was used $(p<0.001)$, and average total usage $(p<0.001)$.

Subgroup analyses between children who are obese or have underlying medical conditions revealed no significant differences with any PAP variables after adjusting for the number of days of PAP download data collection prior to the lockdown (Supplementary e-Table 1). Moreover, there were also no significant differences with the degree of change of PAP usage in the obese group compared to the children with underlying medical 
Table 1 Baseline demographic and clinical characteristics of the study population

\begin{tabular}{ll}
\hline Characteristics & Participants $(N=151)$ \\
\hline Age (years) & $12.6 \pm 4.1$ \\
Male, $n(\%)$ & $92(60.9)$ \\
Weight $(\mathrm{kg})$ & $65.1 \pm 42.4$ \\
Height $(\mathrm{cm})$ & $142.4 \pm 26.2$ \\
Body mass index $\left(\mathrm{kg} / \mathrm{m}^{2}\right)$ & $28.7 \pm 12.4$ \\
Duration of non-invasive ventilation (years) & $3.3 \pm 2.7$ \\
Previous adenotonsillectomy, $n(\%)$ & $88(58.3)$ \\
Independent PAP users, $n(\%)$ & $52(34.4)$ \\
Race & \\
Caucasian/White, $n(\%)$ & $57(37.7)$ \\
$\quad$ Asian, $n(\%)$ & $32(21.2)$ \\
African-American/Black, $n(\%)$ & $23(15.2)$ \\
Others (e.g., mixed, Native, Hispanic), $n$ & $22(14.6)$ \\
$\quad(\%)$ & $17(11.3)$ \\
Did not self-identify, $n(\%)$ & \\
Diagnosis & $96(63.6)$ \\
Underlying medical conditions, $n(\%)$ & $53(35.1)$ \\
Obesity, $n(\%)$ & $2(1.3)$ \\
Healthy, $n(\%)$ & $109(72.2)$ \\
Type of sleep-disordered breathing & $35(23.2)$ \\
OSA only, $n(\%)$ & $4(2.6)$ \\
OSA and/or CSA with nocturnal hypoven- & $3(2.0)$ \\
$\quad$ tilation & \\
Nocturnal hypoventilation only, $n(\%)$ & $49(32.5)$ \\
CSA only, $n(\%)$ & \\
Type of non-invasive ventilation & \\
CPAP, $n(\%)$ & Bi-level PAP, $n(\%)$ \\
\hline
\end{tabular}

Data presented as mean \pm SD unless otherwise indicated

CPAP continuous positive airway pressure, CSA central sleep apnea, $O S A$ obstructive sleep apnea, $P A P$ positive airway pressure conditions across both time periods (data not shown). Among dependent and independent PAP users, independent users were significantly older $(14.5 \pm 2.7$ vs $11.6 \pm 4.4 ; p<0.001)$ with higher BMI $(36.0 \pm 13.7 \mathrm{vs}$ $24.8 \pm 9.6 ; p<0.001$ ), but there were no significant differences with any PAP variables after adjusting for the number of days of PAP download data collection prior to the lockdown. Further, no significant differences were found with changes of PAP usage across all PAP adherence parameters compared to independent users (data not shown). There were also no significant differences found with absolute or percent changes in PAP adherence between (1) males or females or (2) children in different age groups, categorized as (i) 12 years and younger, (ii) between 12 and 15 years old, or (iii) 15 years and older across both time periods (Supplementary e-Table 2 and e-Table 3 , respectively).

Data from the hierarchal multiple regression models with the percentage of days with at least 4-h PAP usage during the lockdown as the dependent variable are shown in Table 5. Baseline characteristics that can affect PAP use such as age (years), independent user (yes or no), diagnosis (obese or other medical conditions), sex (male or female), and PAP type (CPAP or bi-level PAP) as well as the number of days of PAP download data collection prior to the lockdown were included in the regression model, and a significant association was found with diagnosis. Specifically, those with obesity were more likely to demonstrate a decrease in the percentage of days with 4-h PAP usage compared to those with underlying medical conditions $(\beta=-16.64, p=0.02)$. However, using the same model, no significant associations were found with average nightly usage, the number of days of PAP download data collection prior to lockdown, or any baseline characteristic including medical diagnosis $(p=0.06)$.

Table 2 Positive airway pressure data before and during COVID pandemic

\begin{tabular}{|c|c|c|c|}
\hline & Before the COVID-19 lockdown & $\begin{array}{l}\text { During the COVID-19 } \\
\text { lockdown }\end{array}$ & $p$ value \\
\hline Number of days of PAP download data collection & $87 \pm 12$ days & $89 \pm 5$ days & $0.02^{\mathrm{a}}$ \\
\hline Percentage of days of PAP use (\%) & $76.0(36.0-93.3)$ & $86.7(36.0-100.0)$ & $0.03^{\mathrm{a}}$ \\
\hline Percentage of days with $\geq 4$-h PAP usage (\%) & $62.0(15.5-89.0)$ & $76.7(19.0-94.0)$ & $0.02^{\mathrm{a}}$ \\
\hline Average nightly usage (min) & $367.0(218.0-496.0)$ & $406.0(244.0-525.0)$ & $0.006^{\mathrm{a}}$ \\
\hline Average total usage (min) & $274.0(95.0-401.0)$ & $300.0(66.5-443.0)$ & $0.03^{\mathrm{a}}$ \\
\hline
\end{tabular}

The number of days of PAP download data collection is presented as mean $( \pm \mathrm{SD})$. Data presented for $N=151$ as median $\left(25^{\text {th }}-75^{\text {th }}\right.$ percentile $)$ unless otherwise indicated. $p$ values were calculated using the Wilcoxon signed-rank test or paired $t$ test based on the data distribution. Percentage of days of PAP use, overall percentage of days that PAP was used regardless of duration of usage; percentage of days with $\geq 4$-h PAP usage, percentage of days where PAP was used for $\geq 4$-h usage; average nightly usage, average minutes of PAP use based on days where PAP was used; average total usage, average minutes of PAP use based on all the days over which the data was collected

$P A P$ positive airway pressure

${ }^{a} p \leq 0.05$ represents a statistically significant difference before and during the pandemic 
Table 3 Comparison of baseline characteristics between children who showed an increase and those who showed a decrease in positive airway pressure use

\begin{tabular}{|c|c|c|c|}
\hline Characteristics & $\begin{array}{l}\text { Increased PAP usage group } \\
(N=95)\end{array}$ & $\begin{array}{l}\text { Decreased PAP usage group } \\
(N=56)\end{array}$ & $p$ value \\
\hline Age (years) & $13.4(9.6-16.1)$ & $12.4(9.5-16.0)$ & 0.79 \\
\hline Male, $n(\%)$ & $57(60.0)$ & $35(62.5)$ & 0.77 \\
\hline Weight $(\mathrm{kg})$ & $57.8(30.5-95.3)$ & $56.6(21.5-96.6)$ & 0.78 \\
\hline Height $(\mathrm{cm})$ & $150.0(130.0-163.0)$ & $150.0(118.5-165.0)$ & 0.94 \\
\hline Body mass index $\left(\mathrm{kg} / \mathrm{m}^{2}\right)$ & $25.0(18.2-36.0)$ & $27.3(18.9-36.6)$ & 0.94 \\
\hline Duration of non-invasive ventilation (years) & $2.8(1.3-4.3)$ & $2.6(1.2-4.1)$ & 0.64 \\
\hline Previous adenotonsillectomy, $n(\%)$ & $56(58.9)$ & $32(57.1)$ & 0.83 \\
\hline Independent PAP users, $n(\%)$ & $32(33.7)$ & $20(35.7)$ & 0.80 \\
\hline \multicolumn{4}{|l|}{ Ethnicity } \\
\hline Caucasian/White, $n(\%)$ & $23(41.1)$ & $34(35.8)$ & \multirow[t]{5}{*}{0.67} \\
\hline Asian, $n(\%)$ & $11(19.6)$ & $21(22.1)$ & \\
\hline African-American/Black, $n(\%)$ & $7(12.5)$ & $16(16.8)$ & \\
\hline Others (e.g., mixed, Native, Hispanic), $n(\%)$ & $10(17.9)$ & $12(12.6)$ & \\
\hline Did not self-identify, $n(\%)$ & $5(8.9)$ & $12(12.6)$ & \\
\hline \multicolumn{4}{|l|}{ Diagnosis } \\
\hline Underlying medical conditions, $n(\%)$ & $35(62.5)$ & $61(64.2)$ & \multirow[t]{3}{*}{0.99} \\
\hline Obesity, $n(\%)$ & $20(35.7)$ & $33(34.7)$ & \\
\hline Healthy, $n(\%)$ & $1(1.8)$ & $1(1.1)$ & \\
\hline \multicolumn{4}{|l|}{ Type of sleep-disordered breathing } \\
\hline OSA only, $n(\%)$ & $39(69.6)$ & $70(73.7)$ & \multirow[t]{4}{*}{0.10} \\
\hline OSA and/or CSA with nocturnal hypoventilation, $n(\%)$ & $17(30.4)$ & $18(18.9)$ & \\
\hline Nocturnal hypoventilation only, $n(\%)$ & $0(0)$ & $4(4.2)$ & \\
\hline CSA only, $n(\%)$ & $0(0)$ & $3(3.2)$ & \\
\hline \multicolumn{4}{|l|}{ Type of non-invasive ventilation } \\
\hline CPAP, $n(\%)$ & $37(66.1)$ & $65(68.4)$ & \multirow[t]{2}{*}{0.77} \\
\hline Bi-level PAP, $n(\%)$ & $19(33.9)$ & $30(31.6)$ & \\
\hline
\end{tabular}

Data presented for $N=151$ as median $\left(25^{\text {th }}-75^{\text {th }}\right.$ percentile $)$ unless otherwise indicated. $p$ values were calculated using the chi-square test for categorical variables and the Mann-Whitney $U$ test for continuous variables

$C P A P$ continuous positive airway pressure, $P A P$ positive airway pressure

\section{Discussion}

The principal findings of our study were that $63 \%$ of children prescribed PAP prior to the COVID pandemic demonstrated improved PAP adherence during the COVID-19 lockdown. Specifically, we found a $10.6 \%$ increase in adherence from a median of $367.0 \mathrm{~min}$ per night prior to lockdown to $406 \mathrm{~min}$ per night based on days where PAP was used during the lockdown period. Of concern is that $37 \%$ of children in this cohort demonstrated decreased PAP use and 7\% of children abruptly terminated PAP use during the COVID-19 lockdown.

To date, there are no data on changes in PAP usage in children with SDB during the pandemic. Our findings of overall improvement in PAP adherence, based on percentage of days that PAP was used for $\geq 4$-h usage and average nightly usage, are similar to those reported in an adult population. In a study of 7485 adults in France, PAP adherence improved during a 1-month period of the COVID-19 lockdown from an average of 386 min per night prior to the lockdown to $401 \mathrm{~min}$ per night during the lockdown, resulting in a 3.9\% increase in adherence [24]. Possible reasons for the increased PAP use include the fear of being hospitalized and the absence of occupational stress, resulting in increased sleep duration that may enhance PAP adherence [24].

Although there have been concerns among healthcare professionals, patients, and families regarding AGMP and the risk of spreading COVID-19 with use of PAP therapy, our study was reassuring with the majority showing increased PAP usage although the reasons for this are unclear. It is possible that flexible and longer sleep schedules due to online schooling and the lack of need to travel to school may have resulted in increased sleep duration and, consequently, increased PAP usage [25-28]. Moreover, for those children who were dependent on caregivers for PAP usage, where caregivers were continuously at home during this time 
Table 4 Changes in the positive airway pressure adherence data prior to and during the lockdown in the increased and decreased PAP usage groups

\begin{tabular}{|c|c|c|c|c|}
\hline \multirow{2}{*}{$\begin{array}{l}\text { Parameters } \\
\text { PAP adherence data before lockdown }\end{array}$} & \multirow[t]{2}{*}{$\begin{array}{l}\text { Increased PAP usage } \\
\text { group }(N=95)\end{array}$} & \multicolumn{2}{|c|}{ Decreased PAP usage group $(N=56)$} & \multirow[t]{2}{*}{$p$ value } \\
\hline & & & & \\
\hline Percentage of days of PAP use (\%) & $78.0(53.0-96.0)$ & $50.5(18.2-92.5)$ & & $0.02^{\mathrm{a}}$ \\
\hline Percentage of days with $\geq 4$-h PAP usage (\%) & $64.0(30.0-89.0)$ & $38.2(7.2-88.0)$ & & $0.02^{\mathrm{a}}$ \\
\hline Average nightly usage (min) & $386.0(254.0-512.0)$ & $356.5(133.8-470.0)$ & & 0.17 \\
\hline Average total usage (min) & $315.0(147.5-437.0)$ & $104.0(43.5-354.3)$ & & $0.002^{\mathrm{a}}$ \\
\hline \multicolumn{5}{|l|}{ Change in PAP adherence data during lockdown } \\
\hline Absolute change in percentage of days of PAP use (\%) & $8.0(0.0-17.0)$ & $-2.7(-17.8$ to 1.1$)$ & $<0.001^{\mathrm{a}}$ & \\
\hline Absolute change in percentage of days with $\geq 4$-h PAP usage $(\%)$ & $8.0(0.0-22.0)$ & $-5.3(-12.0$ to 0.0$)$ & $<0.001^{\mathrm{a}}$ & \\
\hline Absolute change in average nightly usage (min) & $59.0(24.0-113.0)$ & $-56.5(-104.8$ to -27.0$)$ & $<0.001^{\mathrm{a}}$ & \\
\hline Absolute change in average total usage (min) & $49.0(7.0-117.0)$ & $-30.0(-72.5$ to -2.8$)$ & $<0.001^{\mathrm{a}}$ & \\
\hline Percent change in percentage of days of PAP use & $10.1(0.0-33.6)$ & $-13.0(-89.8$ to 1.8$)$ & $<0.001^{\mathrm{a}}$ & \\
\hline Percent change in percentage of days with $\geq 4$-h PAP usage & $12.7(0.0-50.0)$ & $-28.1(-90.9$ to 1.2$)$ & $<0.001^{\mathrm{a}}$ & \\
\hline Percent change in average nightly usage & $15.2(6.0-36.5)$ & $-22.2(-87.3$ to -10.1$)$ & $<0.001^{\mathrm{a}}$ & \\
\hline Percent change in average total usage & $16.4(2.8-70.1)$ & $-45.8(-94.9$ to -4.22$)$ & $<0.001^{\mathrm{a}}$ & \\
\hline
\end{tabular}

$p$ values were calculated using the Mann-Whitney $U$ test. Percentage of days of PAP use, overall percentage of days that PAP was used regardless of duration of usage; percentage of days with $\geq 4$-h PAP usage, percentage of days where PAP was used for $\geq 4$-h usage; average nightly usage, average minutes of PAP use based on days where PAP was used; average total usage, average minutes of PAP use based on all the days over which the data was collected

$P A P$ positive airway pressure

${ }^{\mathrm{a}} p \leq 0.05$ represents a statistically significant difference across groups

period, may have allowed for longer PAP usage with monitoring by families extending later into the morning.

Although we do not have sleep schedule data available in our study, a cross-sectional study of 593 children, aged 3 to 16 years old, observed longer daily sleep duration in children, as reported by parents, where average sleep duration increased from 9.01 to $9.63 \mathrm{~h} 1$ month after the lockdown. Although children went to sleep later by an average of $0.65 \mathrm{~h}$, increases in sleep duration were attributed to the delayed wake-up time of $1.27 \mathrm{~h}$ [26]. Similarly, in a smaller study with 41 children with obesity aged 6-18 years old, Pietrobelli et al. [28] conducted a telephone interview prior to and 3 weeks following a mandatory lockdown in Italy due to the COVID-19 pandemic and reported an increase in sleep duration by an average of $0.65 \mathrm{~h} /$ day during the lockdown. Further, an online survey completed by 1472 Canadian parents of children aged 5-17 years old, during the first month of the WHO declaring the COVID-19 pandemic, revealed that parents reported an overall increase in sleep duration during the lockdown but no quantitative data were available [27].

It is also important to consider why PAP usage decreased in $37 \%$ of our cohort. It is possible there was a lack of perceived benefits with using PAP [29]. Specifically, older children and adolescents may have adhered more to sleeping schedules that reflect their inherent biologically delayed
Table 5 Multiple regression analysis assessing the factors associated with the percentage of days with a minimum 4-h usage of positive airway pressure during the COVID-19 lockdown

\begin{tabular}{llll}
\hline Parameters & Coefficients & Standard error & $p$ value \\
\hline Age & -0.01 & 0.79 & 0.99 \\
Independent user & -9.16 & 7.04 & 0.20 \\
Diagnosis (e.g., obesity or underlying medical conditions) & -16.64 & 7.02 & $0.02^{\mathrm{a}}$ \\
Sex (male or female) & 9.18 & 6.26 & 0.15 \\
PAP type (e.g., CPAP or bi-level PAP) & 4.24 & 6.60 & 0.52 \\
Number of days of PAP download data collection prior to & 0.06 & 0.05 & 0.21 \\
$\quad$ the lockdown & & &
\end{tabular}

$p$ values were calculated using linear regression. Model shows a significant association with obesity, suggesting that children with obesity were more likely to demonstrate a decrease in the percentage of days with 4-h PAP usage compared to those with underlying medical conditions $(p=0.02)$

$C P A P$ continuous positive airway pressure, $P A P$ positive airway pressure ${ }^{\mathrm{a}} p \leq 0.05$ represents statistical significance 
circadian rhythm during the lockdown, resulting in longer sleep duration and, consequently, a reduction in daytime symptoms such as fatigue and sleepiness [25, 30]. Further, social distancing and social isolation have disrupted daily routines among children, which may have added to their stress and a reluctance to engage in PAP use [30]. Moreover, obese children may be at increased risk of lower PAP usage compared with children with underlying medical conditions, who may be more motivated to use PAP as they perceive greater disease-related risk and the knowledge of PAP-related benefits.

These data highlight the importance of early recognition of children who may decrease or stop PAP use so that clinicians can provide counseling regarding the importance of using PAP and promotion of optimal sleep habits and/or other targeted behavioral interventions as clinically indicated to prevent further decline in PAP use.

The strengths of this study include the objective data obtained from PAP devices which have been validated to be accurate [18]. Further, the diversity and heterogeneity of our sample suggests that these results are likely to be generalizable across pediatric cohorts of children with SDB prescribed PAP therapy.

However, there are some limitations in our study that require consideration. This is a retrospective cohort study, and important data including sleep habits, particularly bed time, awake time, and duration of sleep were not available. We also could not ascertain the reasons for increased or decreased PAP usage as we did not collect qualitative or survey data which would have additionally allowed us to assess the association between concerns using PAP during the COVID-19 pandemic and objective PAP use. Additionally, we did not have data available on whether children or their family members were diagnosed with COVID-19 which may have influenced PAP usage. Finally, PAP adherence was only monitored over a maximum time frame of 6 months and during a time frame when children were not attending schools in person. Easing of lockdown restrictions, return to school, and subsequent changes in schedules of children and their caregivers in September 2020 may have resulted in additional changes in PAP usage that were not captured in this study. Given that significant increases in PAP usage over a short time frame are not common, further research is needed to identify the behavioral modifications that occurred during the COVID-19 pandemic that could be leveraged and applied to optimize clinical care in children with SDB using PAP. Moreover, larger prospective longitudinal studies are needed to ascertain whether the changes in PAP adherence are sustained beyond the defined time periods in this study and particularly beyond the COVID-19 pandemic.

\section{Conclusion}

COVID-19 pandemic continues to have a significant impact worldwide including an impact on sleep in children and caregivers. Although this study reassuringly demonstrates that $63 \%$ of children with SDB showed increased PAP adherence, children with known suboptimal PAP usage showed further decreases in PAP usage during the COVID-19 pandemic. Such information is beneficial for clinicians to recognize and provide targeted therapeutic interventions to reinforce adherence to PAP in the pediatric population during prolonged lockdown periods. Further, in order to improve sleep health and PAP adherence among children with SDB, there is an urgent need to explore the facilitators and barriers to both increasing the number of hours of sleep and PAP usage in the pediatric population both during and beyond the COVID-19 pandemic.

Supplementary Information The online version contains supplementary material available at https://doi.org/10.1007/s11325-021-02409-w.

Abbreviations AGMP, Aerosol-generating medical procedure; AT, Adenotonsillectomy; Bi-level PAP, Bi-level positive airway pressure; BMI, Body mass index; CPAP, Continuous positive airway pressure; CSA, Central sleep apnea; etCO $\mathrm{O}_{2}$, End-tidal $\mathrm{CO}_{2}$, IQR, Interquartile range; OSA, Obstructive sleep apnea; PAP, Positive airway pressure; SD, Standard deviation; SDB, Sleep-disordered breathing; tcCO $\mathrm{CO}_{2}$ Transcutaneous $\mathrm{CO}_{2}$; WHO, World Health Organization

Acknowledgements The study was conducted at The Hospital for Sick Children (SickKids) in Toronto, Canada.

Author contribution KS, SS, and IN conceived and designed the study. $\mathrm{KS}, \mathrm{AB}$, and MT collected the study data. SS analyzed the data. KS, $\mathrm{SS}$, and IN interpreted the study results. KS and SS drafted the manuscript. KS, SS, GV, DB, AB, MT, and IN edited, revised, and approved the version of the manuscript to be published.

Funding This work was supported by funds from the Department of Paediatrics at The Hospital for Sick Children, Toronto, Canada, and the Canadian Institutes of Health Research through the Canadian Sleep and Circadian Network, Canada.

Data availability All data relevant to the study are included in the article or uploaded as supplementary information.

\section{Declarations}

Ethics approval This study was approved by the SickKids Research Ethics Board (REB No. 1000071964).

Conflict of interest The authors declare no competing interests. 


\section{References}

1. Kaditis AG, Alonso Alvarez ML, Boudewyns A, Alexopoulos EI, Ersu R, Joosten K, Larramona H, Miano S, Narang I, Trang H, Tsaoussoglou M, Vandenbussche N, Villa MP, Van Waardenburg D, Weber S, Verhulst $S$ (2016) Obstructive sleep disordered breathing in 2- to 18-yearold children: diagnosis and management. Eur Respir J 47(1):69-94. https://doi.org/10.1183/13993003.00385-2015

2. Lumeng JC, Chervin RD (2008) Epidemiology of pediatric obstructive sleep apnea. Proc Am Thorac Soc 5(2):242-252. https://doi.org/10.1513/pats.200708-135MG

3. Narang I, Mathew JL (2012) Childhood obesity and obstructive sleep apnea. J Nutr Metab 2012:134202. https://doi.org/10.1155/2012/134202

4. Lee CF, Lee CH, Hsueh WY, Lin MT, Kang KT (2018) Prevalence of obstructive sleep apnea in children with down syndrome: a meta-analysis. J Clin Sleep Med 14(5):867-875. https://doi.org/10.5664/jcsm.7126

5. Amin R, Sayal P, Sayal A, Massicote C, Pham R, Al-Saleh S, Drake J, Narang I (2015) The association between sleep-disordered breathing and magnetic resonance imaging findings in a pediatric cohort with Chiari 1 malformation. Can Respir J 22(1):31-36. https://doi.org/10.1155/2015/831569

6. McLaren AT, Bin-Hasan S, Narang I (2019) Diagnosis, management and pathophysiology of central sleep apnea in children. Paediatr Respir Rev 30:49-57. https://doi.org/10.1016/j.prrv.2018.07.005

7. Beebe DW (2006) Neurobehavioral morbidity associated with disordered breathing during sleep in children: a comprehensive review. Sleep 29(9):1115-1134

8. Galland B, Spruyt K, Dawes P, McDowall PS, Elder D, Schaughency $E$ (2015) Sleep disordered breathing and academic performance: a meta-analysis. Pediatrics 136(4):e934-946. https://doi. org/10.1542/peds.2015-1677

9. Narang I, McCrindle BW, Manlhiot C, Lu Z, Al-Saleh S, Birken CS, Hamilton J (2018) Intermittent nocturnal hypoxia and metabolic risk in obese adolescents with obstructive sleep apnea. Sleep Breath 22(4):1037-1044. https://doi.org/10.1007/ s11325-018-1631-9

10. Yilmaz E, Sedky K, Bennett DS (2013) The relationship between depressive symptoms and obstructive sleep apnea in pediatric populations: a meta-analysis. J Clin Sleep Med 9(11):1213-1220. https://doi.org/10.5664/jcsm.3178

11. Amin R, Holler T, Narang I, Cushing SL, Propst EJ, Al-Saleh S (2018) Adenotonsillectomy for obstructive sleep apnea in children with complex chronic conditions. Otolaryngol Head Neck Surg 158(4):760-766. https://doi.org/10.1177/0194599817746959

12. Marcus CL, Brooks LJ, Draper KA, Gozal D, Halbower AC, Jones J, Schechter MS, Sheldon SH, Spruyt K, Ward SD, Lehmann C, Shiffman RN (2012) Diagnosis and management of childhood obstructive sleep apnea syndrome. Pediatrics 130(3):576-584. https://doi.org/10.1542/peds.2012-1671

13. Amin R, Al-Saleh S, Narang I (2016) Domiciliary noninvasive positive airway pressure therapy in children. Pediatr Pulmonol 51(4):335-348. https://doi.org/10.1002/ppul.23353

14. Parmar A, Baker A, Narang I (2019) Positive airway pressure in pediatric obstructive sleep apnea. Paediatr Respir Rev 31:43-51. https://doi.org/10.1016/j.prrv.2019.04.006

15. Marcus CL, Radcliffe J, Konstantinopoulou S, Beck SE, Cornaglia MA, Traylor J, DiFeo N, Karamessinis LR, Gallagher PR, Meltzer LJ (2012) Effects of positive airway pressure therapy on neurobehavioral outcomes in children with obstructive sleep apnea. Am J Respir Crit Care Med 185(9):998-1003. https://doi.org/10.1164/ rccm.201112-2167OC

16. DelRosso LM, King J, Ferri R (2018) Systolic blood pressure elevation in children with obstructive sleep apnea is improved with positive airway pressure use. J Pediatr 195:102-107.e101. https://doi.org/10.1016/j.jpeds.2017.11.043
17. Ramirez A, Khirani S, Aloui S, Delord V, Borel JC, Pepin JL, Fauroux B (2013) Continuous positive airway pressure and noninvasive ventilation adherence in children. Sleep Med 14(12):1290 1294. https://doi.org/10.1016/j.sleep.2013.06.020

18. Bhattacharjee R, Benjafield AV, Armitstead J, Cistulli PA, Nunez CM, Pepin J-LD, Woehrle H, Yan Y, Malhotra A (2020) Adherence in children using positive airway pressure therapy: a big-data analysis. The Lancet Digital Health 2(2):e94-e101. https://doi. org/10.1016/S2589-7500(19)30214-6

19. World Health Organization (2020) Rational use of personal protective equipment (PPE) for coronavirus disease (COVID19). https://apps.who.int/iris/bitstream/handle/10665/331498/ WHO-2019-nCoV-IPCPPE_use-2020.2-eng.pdf

20. Ayas NT, Fraser KL, Giannouli E, Hanly PJ, Kendzerska T, Katz SL, Lachmann BN, Lajoie A, Minville C, Morrison D, Narang I, Povitz M, Skomro R, Spurr KF (2020) Key highlights from the Canadian Thoracic Society's position statement on optimizing the management of sleep disordered breathing during the coronavirus disease 2019 pandemic. Chest 158(3):899-900. https://doi.org/10. 1016/j.chest.2020.05.572

21. Barker J, Oyefeso O, Koeckerling D, Mudalige NL, Pan D (2020) COVID-19: community CPAP and NIV should be stopped unless medically necessary to support life. Thorax 75(5):367. https://doi. org/10.1136/thoraxjnl-2020-214890

22. Centers for Disease Control and Prevention (2016) Defining childhood obesity. https://www.cdc.gov/obesity/childhood/defining.html

23. ResMed AirView. https://www.resmed.com/epn/en/healthcareprofessional/products/monitoring-and-data-management/airvi ew-patient-management-system.html. Accessed Nov 22, 2020

24. Attias D, Pepin JL, Pathak A (2020) Impact of COVID-19 lockdown on adherence to continuous positive airway pressure by obstructive sleep apnoea patients. Eur Respir J 56(1). https://doi. org/10.1183/13993003.01607-2020

25. Bates LC, Zieff G, Stanford K, Moore JB, Kerr ZY, Hanson ED, Barone Gibbs B, Kline CE, Stoner L (2020) COVID-19 impact on behaviors across the 24-hour day in children and adolescents: physical activity, sedentary behavior, and sleep. Children (Basel) 7(9). https://doi.org/10.3390/children7090138

26. Lim MTC, Ramamurthy MB, Aishworiya R, Rajgor DD, Tran AP, Hiriyur P, Kunaseelan S, Jabri M, Goh DYT (2020) School closure during the coronavirus disease 2019 (COVID-19) pandemic - impact on children's sleep. Sleep Med 78:108-114. https://doi. org/10.1016/j.sleep.2020.12.025

27. Moore SA, Faulkner G, Rhodes RE, Brussoni M, Chulak-Bozzer T, Ferguson LJ, Mitra R, O'Reilly N, Spence JC, Vanderloo LM, Tremblay MS (2020) Impact of the COVID-19 virus outbreak on movement and play behaviours of Canadian children and youth: a national survey. Int J Behav Nutr Phys Act 17(1):85. https://doi. org/10.1186/s12966-020-00987-8

28. Pietrobelli A, Pecoraro L, Ferruzzi A, Heo M, Faith M, Zoller T, Antoniazzi F, Piacentini G, Fearnbach SN, Heymsfield SB (2020) Effects of COVID-19 lockdown on lifestyle behaviors in children with obesity living in Verona, Italy: a longitudinal study. Obesity (Silver Spring, Md) 28(8):1382-1385. https://doi.org/10.1002/oby.22861

29 Alebraheem Z, Toulany A, Baker A, Christian J, Narang I (2018) Facilitators and barriers to positive airway pressure adherence for adolescents. A qualitative study. Ann Am Thorac Soc 15(1):8388. https://doi.org/10.1513/AnnalsATS.201706-472OC

30. Naime S, Weiss M, Nino G (2020) Effects of COVID-19 pandemic on adherence to obstructive sleep apnea therapy: a case report. Clin Case Rep 9(1):12-14. https://doi.org/10.1002/ccr3.3363

Publisher's note Springer Nature remains neutral with regard to jurisdictional claims in published maps and institutional affiliations. 